\title{
Experimental prototype of a novel ring resonator-based optical beamformer system
}

\author{
L. Zhuang*, A. Meijerink*, C. G. H. Roeloffzen*, D. A. I. Marpaung*, \\ R. G. Heideman ${ }^{\dagger}$, M. Hoekman ${ }^{\dagger}$, A. Borreman ${ }^{\dagger}$, D. H. Geuzebroek ${ }^{\dagger}$, A. Leinse ${ }^{\dagger}$, and W. van Etten* \\ ${ }^{*}$ University of Twente, Faculty of Electrical Engineering, Mathematics and Computer Science, \\ Telecommunication Engineering group, P.O. Box 217, 7500 AE Enschede, The Netherlands \\ ${ }^{\dagger}$ LioniX B.V., P.O. Box 456, 7500 AH, Enschede, The Netherlands \\ Email: 1.zhuang@ewi.utwente.nl
}

\begin{abstract}
A novel optical beamformer system for phased array transmit antennas is proposed and experimentally demonstrated. It is based on an optical IM/DD link through a ring resonator-based optical beam forming network chip.
\end{abstract}

\section{INTRODUCTION}

Implementing a beamformer circuit for phased array antennas in the optical domain offers several advantages like compactness, small weight, low loss, frequency independence, high bandwidth, and EMI immunity. Most known approaches are based on optical phase shifters [1] or switchable true time delay (TTD) arrays [2], having the disadvantage of beam squint or limited tuning resolution, respectively. An alternative that does offer both continuous tunability and TTD is based on chirped fibre gratings (CFGs) [3], but this has the disadvantage of requiring bulky optical components and a tunable laser.

In this paper a squint-free, continuously tunable beamformer mechanism for a phased array transmission system is proposed that does not require a tunable laser. It is based on a fully integrated optical beam forming network (OBFN) using cascades of optical ring resonators (ORRs) as tunable delay elements. Its principles are explained in Section II, and in Section III measurements are presented on an experimental prototype of a full beamformer system, based on a $1 \times 2$ OBFN. Conclusions are formulated in Section IV.

\section{PRINCIPLES}

A narrowband continuously tunable optical TTD device can be realized as a recirculating waveguide coupled to a straight waveguide [4]-[7]. In the ideal (lossless) case this straight waveguide will then behave as an all-pass filter with a periodic, bell-shaped group delay response, as illustrated by the dashed lines in Fig. 1. The maximum group delay occurs at the resonance frequency $f_{i}$, which can be varied by tuning the round-trip phase shift $\phi_{i}$ of the ORR. The maximum delay can be varied by tuning the coupling coefficient $\kappa_{i}$ between waveguide and ORR. The bell shape has a constant area, so there is a trade-off between peak delay and optical bandwidth.

A larger delay-bandwidth product can be achieved by cascading multiple ORR sections, as shown in the inset of Fig. 1. The total group delay response of the cascade follows by summing the individual group delay responses, resulting in the

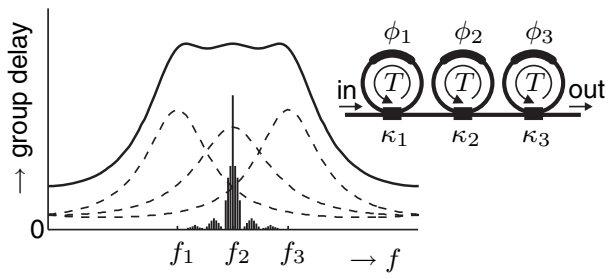

Fig. 1. Theoretical group delay response of three cascaded ORRs with resonance frequencies $f_{i}$. The thin lines represent the spectrum of an optical carrier modulated by a pulse signal. (Inset: three ORR sections in series, with round-trip time $T$, round-trip phase shifts $\phi_{i}$ and coupling coefficients $\kappa_{i}$ )

solid line. It can be flattened by tuning the ORRs to different resonance frequencies. Such multi-stage delay element has a trade-off between peak delay, optical bandwidth, relative delay ripple and number of ORR sections [4]-[7].

A complete OBFN can be obtained by grouping several delay and combining elements in one optical circuit. In [6] we presented the - to our knowledge- first single-chip realization of such an OBFN, based on the $1 \times 4$ binary tree topology shown in Fig. 2. The chip was fabricated in a CMOS-compatible waveguide technology of LioniX BV, called TriPleX [8]. The measured optical group delay responses showed good agreement with theory, and were later extended to a $1 \times 8$ OBFN [7].

This paper reports the first full system prototype using this beam forming concept. To keep this first experiment simple, it is based on an OBFN with only two output ports, using the system topology shown in Fig. 3. The light of a laser is externally modulated by a pulsed signal $s(t)$, by means of a quadrature-biased Mach-Zehnder modulator (MZM). The spectrum of the modulated optical signal consists of an optical carrier and two sidebands, as illustrated by the thin line spectrum around $f_{2}$ in Fig. 1. After splitting in the OBFN the optical signals are delayed with respect to each other and converted back to the electrical domain by the detector.

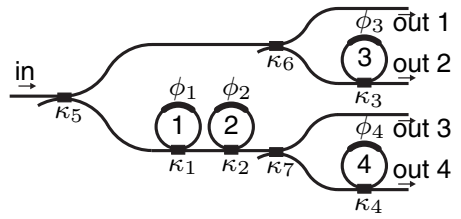

Fig. 2. Binary tree-based $1 \times 4$ OBFN consisting of 4 ORRs and 3 combiners 


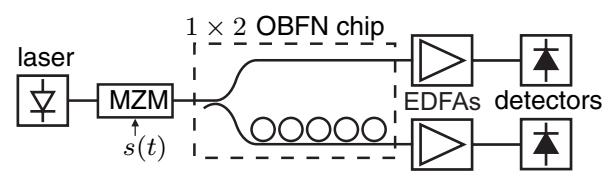

Fig. 3. Beamformer system prototype

\section{EXPERIMENTAL SYSTEM PROTOTYPE}

An experimental prototype of this system was built using a Santec TSL-210 tunable laser, an Axanex PowerLog FA$20 \mathrm{MZM}$, a $1 \times 2 \mathrm{OBFN}$ chip (fabricated by LioniX BV), two Firmstein PR25R Erbium-doped fiber amplifiers (to compensate for the relatively high fiber-chip coupling losses in this particular OBFN chip), and two Emcore R2860E receiver modules. The pulsed input signal $s(t)$ of the MZM is supplied by an HP 8082A pulse generator, and has a period of $80 \mathrm{~ns}$ and a pulse length of 3 ns. The group delay of the lower path in the OBFN is varied by properly changing the optical parameters of the rings. This is done by means of chromium heaters on top of the rings, based on the well-known thermooptical effect. The measured group delay curves (obtained by means of the measurement setup presented in [6], [7], using an Agilent N5230A network analyzer) are shown in Fig. 4(a). For each different setting the detector signals are measured by means of an Agilent 54854A digital sampling oscilloscope. The resulting pulse shapes are shown in Fig. 4(b).

The trace for heater setting 1 in Fig. 4(b) represents the output pulse for zero group delay response of the lower path of the OBFN over about $1 \mathrm{GHz}$ input pulse bandwidth. Besides, this trace equals to the output of the upper path when the power splitter of the OBFN works at $-3 \mathrm{~dB}$ point and signal path lengths are equal for both paths. The upper path is independent of the heater settings and normally used as zero delay reference. The other traces represent the output pulses of the lower path for different heater settings, corresponding to the group delay curves in Fig. 4(a). These traces show that the input pulse is delayed by the ORRs with an increasing delay value (corresponding to the group delay responses over the input pulse bandwidth). Obviously the loss of the lower path in the OBFN increases with increasing delay, due to roughly $0.25 \mathrm{~dB} / \mathrm{cm}$ waveguide losses in the ORRs on this chip. Fig. 4(a) also illustrates the delay-bandwidth tradeoff of the OBFN. The output pulse for higher delay is more significantly distorted due to the fact that the corresponding group delay response (see Fig. 4(a)) does not provide enough bandwidth to accomodate the modulated optical signal (also see Fig. 1) and the delay ripple over the signal band becomes more serious. In the case that the group delay response is too narrow and too sharp for the bandwidth of an input pulse as that for heater setting 5, the effect of pulse splitting can be observed at the output as shown in the inset of Fig. 4(b).

\section{CONCLUSION AND DISCUSSION}

A novel squint-free, continuously tunable beamformer using an integrated ORR-based OBFN has been proposed, and experimentally demonstrated by means of time-domain measurements on baseband pulses. Time delays of a 3-ns pulse

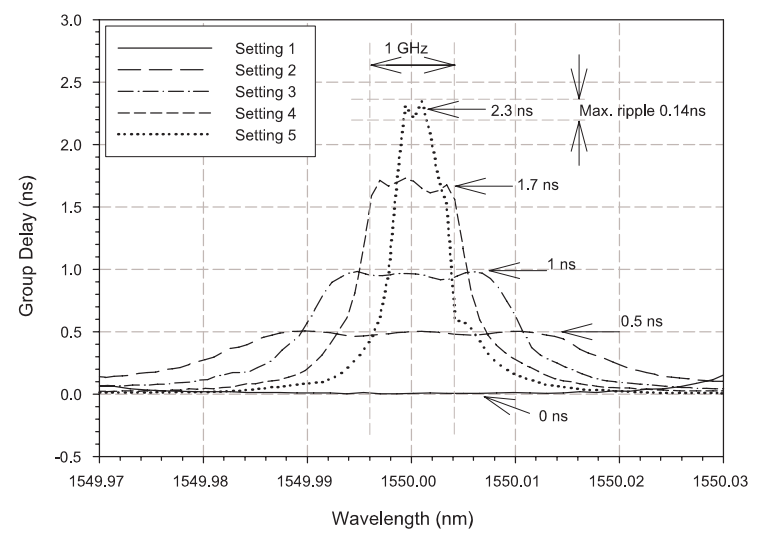

(a) Measured optical group delay responses at the lower output for different heater settings

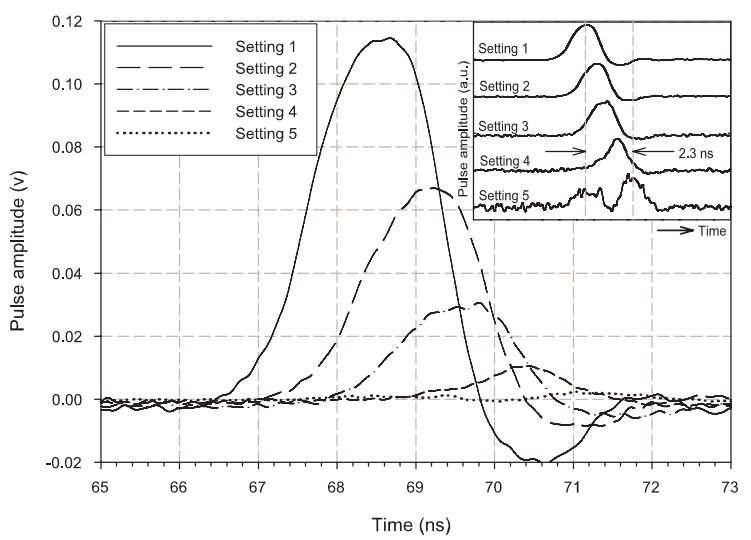

(b) Measured pulse shapes at the lower output (inset: pulse shapes in arbitrary amplitude units for comparison)

Fig. 4. Measurement results

for different group delay responses of a $1 \times 2$ OBFN chip are demonstrated up to $2.3 \mathrm{~ns}$ (69 $\mathrm{cm}$ delay distance in air).

The maximum frequency of $s(t)$ in this system is limited to half the OBFN bandwidth. This can be increased by using optical SSB-SC modulation and balanced detection [9]; the optical bandwidth then equals the bandwidth of $s(t)$. A demonstrator for such topology is currently being built.

\section{ACKNOWLEDGMENT}

This work was part of the Broadband Photonic Beamformer project and the SMART project, which are supported by the Dutch Ministry of Economic Affairs, SenterNovem project numbers IS052081 and IS053030, respectively; the latter project is part of the Eureka PIDEA ${ }^{+}$project SMART.

\section{REFERENCES}

[1] G. Grosskopf et al., IEEE Photon. Technol. Lett., pp. 1169-1171, 2002.

[2] M. A. Piqueras et al., IEEE Trans. MTT, pp. 887-899, 2006.

[3] J. L. Corral et al., Electron. Lett., pp. 209-211, 1998.

[4] G. Lenz et al., IEEE J. Quantum Electron., pp. 525-532, 2001.

[5] M. S. Rasras et al., IEEE Photon. Technol. Lett., pp. 834-836, 2005.

[6] L. Zhuang et al., Proc. MWP'2006, p. F1.4.

[7] L. Zhuang et al., "Single-chip ring resonator-based $1 \times 8$ optical beam forming network in CMOS-compatible waveguide technology," IEEE Photon. Technol. Lett., accepted for publication, 2007.

[8] R. G. Heideman et al., Proc. ECIO'2007, p. WB0.

[9] A. Meijerink et al., Proc. IEEE Benelux SCVT'2006, pp. 7-12. 